\section{HISTORIA DE LA REVISTA EDUCACIÓN FÍSICA-CHILE: APROXIMACIÓN BIBLIOMÉTRICA (1929-2013)}

\author{
HISTORY OF THE JOURNAL EDUCACION FISICA-CHILE: A BIBLIOMETRIC \\ APPROACH (1929-2013)
}
HISTÓRIA DA REVISTA EDUCACIÓN FÍSICA-CHILE: APROXIMAÇÃO
BIBLIOMÉTRICA (1929-2013)

Mikel Pérez-Gutiérrez*, Carlos Gutiérrez-García**

\author{
(MelPerezutherrez, Carlos Gutiertez-Garcia*
}

Palabras-clave Bibliometría. Historia. Deportes. Chile.

\section{Keywords:} Bibliometrics. History. Sports. Chile.

\section{Palavras chave} Bibliometria. História. Esportes. Chile.

\begin{abstract}
Resumen: El objetivo de este trabajo fue realizar un análisis histórico-bibliométrico de la revista Educación Física-Chile desde 1929 hasta 2013. Se consultaron todos los artículos publicados en la revista, registrando sus datos bibliográficos, institución, país y temática principal para, posteriormente, proceder a su análisis. Los resultados muestran que Educación Física-Chile ha sido un referente fundamental para la difusión de las investigaciones relacionadas con el conjunto de las Ciencias de la Actividad Física y el Deporte en Chile, y revelan la situación marginal de la revista en la actualidad.
\end{abstract}

Abstract: The aim of this paper was to carry out a historic-bibliometric analysis of the journal Educación Física-Chile from 1929 to 2013. All articles included in that journal were consulted, and their bibliographic data, institutions, countries and main topics were recorded and then analysed. Results showed that Educación Física-Chile hasbeen a key reference for spreading Physical Education and Sport Sciences studies in Chile, although it has now a marginal position in the field.

Resumo: 0 objetivo deste trabalho foi realizar uma análise histórico-bibliométrica da revista Educación Física-Chile de 1929 a 2013. Consultaram-se todos os artigos publicados na revista, registrando-se os dados bibliográficos, a instituição, o país e a temática principal para, posteriormente, se proceder à sua análise. Os resultados mostram que a Educación Física-Chile foi uma referência fundamental para a difusão de investigações relacionadas com o conjunto das Ciências da Atividade Física e Desporto no Chile, e revelam a situação marginal da revista na atualidade.
Universidad Autónoma de Chile. Temuco, Chile.

E mail: mikel.perez@uautonoma.cl

"Universidad de León. León, España. E mail: cgutg@unileon.es

Recebido em: 03.02-2015 Aprovado em: 13-04-2015 (c) (1) (8) Licence 


\section{INTRODUCCIÓN ${ }^{1}$}

La Educación Física en Chile alcanzó su oficialización y los estudios superiores el 6 de marzo de 1906 con la creación del Instituto de Educación Física y Manual al alero de la Universidad de Chile (COFRÉ ILUFFI, 1986, 1989, GUARDA ETCHEVERRY, 2006b). Este hecho constataba la creciente preocupación social y política que había existido hacia las prácticas físicas y gimnásticas desde el siglo XIX (GUARDA ETCHEVERRY, 2006b, RUIZ URBINA, 1956) y daba comienzo a una nueva etapa en la que el Instituto sería un referente no solo para la evolución de la Educación Física a nivel nacional, por formar a los futuros profesores de Educación Física que se irían integrando en las escuelas y liceos a lo largo del país así como a la planta docente de las nuevas facultades y carreras de Educación Física que se crearían en la segunda mitad del s. XX, sino también en el ámbito internacional, siendo sus profesores invitados a dictar cursos y charlas en distintos países latinoamericanos (COFRÉ ILUFFI, 1989, RUIZ URBINA, 1956). Esta condición de referente nacional e internacional se vio reforzada por la aparición en el año 1929, tras 23 años de andadura, de la revista denominada Educación Física siendo considerada la más antigua del mundo de su especialidad (GUARDA ETCHEVERRY, 2006b, SALAS, 1989).

La revista nacía como órgano oficial de la Dirección General de Educación Física y la Escuela de Profesores de Educación Física, siendo actualmente el Departamento de Educación Física, Deportes y Recreación (DEFDER) de la Universidad Metropolitana de Ciencias de la Educación (UMCE). A pesar de que la revista pasó desde aquella fecha por cuatro etapas distintas y ha dependido de diversas instituciones, ha mantenido desde sus inicios una línea editorial centrada en informar de los principales avances, hechos y sucesos acaecidos en torno a la Educación Física, incluyendo para ello todo tipo de documentos, desde artículos científicos hasta crónicas y notas bibliográficas. De esta manera resumía la primera editorial de la revista bajo el título de Boletín de Educación Física, en el año 1934, sus objetivos y carácter:

Este Boletín tratará de mantener a sus lectores al corriente de las novedades que en los principales centros científicos y culturales se vayan produciendo. También dará a conocer el aporte que nuestro Instituto pueda allegar, a la medida de sus fuerzas, a la dilucidación de tales problemas, por medio de los trabajos de experimentación que en sus laboratorios comienzan ya a realizarse. Con tal objeto tendrá una sección científico-técnica de carácter permanente.

Además, nuestro boletín desea ser antena que recoja y dé a conocer los hechos más salientes que se produzcan en el campo de la Educación Física, tanto en el país como en el extranjero; asimismo, informará sobre los libros, revistas, memorias y demás publicaciones sobre la materia. Con tal fin tendrá una sección Informaciones y una sección Bibliográfica." (NOTAS..., 1934, p. 1)

La antigüedad de esta revista junto con el ambicioso proyecto editorial que guiaba su publicación convirtieron a Educación Física-Chile en una de las revistas más importantes de su especialidad, a pesar de que también sufrió problemas económicos que impidieron su edición en determinados periodos y que mermaron su periodicidad. No obstante, han sido varios los trabajos que se han encargado de celebrar sus aniversarios, resumir su trayectoria histórica o incluso analizar parte de sus publicaciones para entender y construir la historia de la Educación Física en Chile. En este sentido, Croxatto (1986) resumía el rol e importancia social de esta 
revista en un ejemplar dedicado a celebrar los ochenta años de existencia del Instituto de Educación Física y Manual.

Por su parte, el trabajo de Salas (1989) esbozó la historia de Educación Física-Chile a través de la labor de sus principales directores, resumiendo los rasgos distintivos de cada etapa y las orientaciones entregadas por cada director. Este trabajo destaca por ser el primero en reconocer que los inicios de la publicación datan de 1929 ya que la revista "estuvo siempre al servicio del Instituto de Estudios Superiores destinado a la formación de profesores de Educación Física" (SALAS, 1989, p. 4).

De manera similar, Guarda Etcheverry (2006a) describió la historia de Educación FísicaChile a través de sus cuatro etapas o denominaciones, centrando nuevamente la atención sobre la dirección de la revista y el equipo de redacción que participó en la edición y publicación de la misma a lo largo de los años. Para ello realizó un análisis detallado de todos los cambios de dirección y edición que hubo en la revista apuntando sus periodos de ejecución, a diferencia del trabajo de Salas (1989) que solo detallaba a los cinco directores más importantes.

Finalmente, Educación Física-Chile también ha sido utilizada como una fuente de información principal para descubrir y construir la historia de la Educación Física en Chile durante el periodo de 1934 a 1962. A través del análisis de las características formales (formato, estructura, objetivos, directores, administradores, precio, anuncios y publicidad) y de contenido (distribución de artículos por año, sección, tema y bloque temático) de la revista, Poblete Gálvez (2014) ha desvelado en su tesis doctoral algunos de los principales hechos históricos además de ofrecer una visión de los principales temas que preocupaban a la sociedad chilena en materia de Educación Física durante el periodo analizado.

A pesar de estas aproximaciones, hasta el momento ningún trabajo se ha encargado de estudiar la historia de la revista Educación Física-Chile desde la perspectiva de las Ciencias de la Documentación. Este tipo de estudios sobre revistas concretas comienza a ser habitual en la literatura científica relacionada con las Ciencias de la Actividad Física y el Deporte (CCAFD), habiéndose analizado revistas como Archivos de Medicina del Deporte (LÓPEZ MORENO; VELASCO MARTÍN, 2004), Citius-Altius-Fortius (PERRINO PEÑA, 2014), European Sport Management Quarterly (PITTS; DANYLCHUK; QUARTERMAN, 2014), Journal of History of Sport (HENNESSEY, 2004), Journal of Sports Sciences (NEVILL; ATKINSON; HUGHES, 2008, WILLIAMS; HARDY; MUTRIE, 2008), o Revista de Psicología del Deporte (ARBINAGA IBARZÁBAL; ARAGÓN; TEJEDOR, 2010), entre otras. El interés de estos trabajos es analizar la evolución de estas revistas, por ser referentes en su disciplina debido a su historia y/o importancia en la difusión de trabajos, y descubrir los aportes que han realizado a su campo científico. Los aspectos analizados en estos estudios son fundamentalmente los autores, instituciones y países más productivos, las temáticas tratadas y el análisis de las citas. Por ello, el objetivo de la presente investigación es realizar un análisis histórico de la revista Educación Física-Chile desde 1929 hasta 2013 desde el ámbito bibliométrico. Además, pretende ofrecer un análisis exhaustivo del periodo de publicación, director, periodo de dirección y afiliación de la revista a lo largo de su historia.

\section{METODOLOGÍA}

Se incluyeron todos los artículos o revisiones publicados en Educación Física-Chile desde 1929 hasta 2013. Para ello, se consultaron directamente todos los números de la revista 
en la biblioteca del DEFDER de la UMCE y en la Biblioteca Nacional de Chile, y se creó un registro bibliográfico de cada referencia de acuerdo a las recomendaciones de la Organización Internacional de Normalización para las referencias bibliográficas (AENOR, 2013) compuesto por autor, año, título, revista, volumen, número y páginas. Estos datos fueron ingresados en el programa gestor de referencias Endnote X6. Además, cada artículo fue clasificado por disciplina científica de acuerdo a las recomendaciones propuestas por Devís et al. (2010) para la clasificación temática y disciplinar de las artículos relacionados con las CCAFD, tras la revisión del título, palabras clave y resumen/introducción de cada documento. Los artículos fueron clasificados de la siguiente manera:

- Actividad física y salud: estudian las consecuencias para la salud derivadas de la práctica de actividad física.

- Antropología: analizan las costumbres y/o cultura deportiva del ser humano.

- Antropometría: estudian las medidas corporales del ser humano.

- Arquitectura: estudian las construcciones deportivas y sus características.

- Biomecánica: analizan las variables cinemáticas y mecánicas en la práctica de la actividad física.

- Cinesiología: profundizan en el análisis y tratamiento de problemas en el movimiento humano.

- Derecho: estudian las leyes y/o normas aplicadas al ámbito de las CCAFD.

- Didáctica: exponen los diferentes métodos de enseñanza de la actividad física.

- Documentación: relacionados con el procesamiento de la información, como por ejemplo la creación de bibliografías.

- Filosofía: profundizan en el sentido y significado de las CCAFD.

- Fisiología: analizan las funciones orgánicas del ser humano.

- Gestión: dedicados a la administración de la actividad física en sus diferentes ámbitos y organizaciones.

- Historia: estudian el pasado de las actividades físicas.

- Informática: relacionados con la computación y su aplicación a la actividad física.

- Medicina del Deporte: estudian el mantenimiento y mejora de la condición y/o rendimiento físico, como por ejemplo las lesiones o el doping.

- Nutrición: dedicados a los procesos alimenticios y la ingesta de nutrientes en su relación con la actividad física.

- Psicología: estudian los procesos psicológicos implicados en la actividad física.

- Psicomotricidad: dedicados al estudio de la interacción cuerpo-mente, como por ejemplo el desarrollo motor o la lateralidad.

- Sociología: profundizan en los aspectos sociales de la actividad física, como por ejemplo los hábitos deportivos.

- Teoría de la Educación Física y el Deporte: dedicados a los fundamentos teóricos de las CCAFD, como por ejemplo la definición de conceptos.

- Teoría del Entrenamiento Deportivo: analizan los procesos de entrenamiento.

Estas disciplinas fueron agrupadas en cuatro grandes ramas de conocimiento (Artes y Humanidades, Ciencias de la Vida, Ciencias Sociales y Tecnología) de acuerdo a la clasificación propuesta para la Web of Science (THOMSON REUTERS, 2012). Posteriormente, los datos 
fueron exportados al programa Excel 2013 para realizar el análisis bibliométrico centrado en la distribución de artículos por periodo, año, disciplina científica, autor, firma, institución y país. Asimismo, se consultaron las portadas, contraportadas, páginas de créditos, editoriales, etc. de las revistas para extraer otras informaciones relevantes de la revista.

\section{RESULTADOS}

En sus 85 años de historia, Educación Física-Chile ha pasado por cuatro etapas que corresponden a sus distintos títulos de cabecera. El Cuadro 1 resume estos periodos, junto con su afiliación, directores y periodos de dirección. La revista no fue publicada entre 19321933, 1970-1973 ni en el año 2010. Aunque su edición ha dependido siempre del mismo instituto/departamento, éste ha estado supeditado a diferentes órganos de gobierno, como la Dirección General de Educación Física, la Universidad de Chile o la UMCE. En total, ha tenido doce directores, siendo Rosauro Salas y Luis Bisquertt los que más permanecieron en el cargo. Por el contrario, la dirección de la revista durante los últimos tres años ha cambiado anualmente.

Cuadro 1 - Etapas de la revista Educación Física-Chile (1929-2013)

\begin{tabular}{|c|c|c|}
\hline Título y periodo & Afiliación & Directores y periodos de dirección \\
\hline $\begin{array}{l}\text { Educación Física } \\
\text { 1929-1931 }\end{array}$ & $\begin{array}{c}\text { Dirección General de Educación } \\
\text { Física / Escuela de Profesores } \\
\text { de Educación Física }\end{array}$ & $\begin{array}{l}\text { Osvaldo Kolbach y Rosauro Salas Arellano } \\
n^{\circ} 1 \text { (Abril 1929) - } n^{\circ} 24 \text { (Abril 1931) }\end{array}$ \\
\hline \multirow{4}{*}{$\begin{array}{c}\text { Boletín de } \\
\text { Educación Física } \\
1934-1950\end{array}$} & \multirow{4}{*}{$\begin{array}{l}\text { Instituto de Educación Física de } \\
\text { la Universidad de Chile }\end{array}$} & 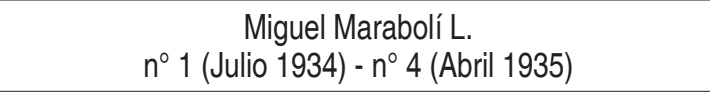 \\
\hline & & $\begin{array}{c}\text { Joaquín Cabezas } \\
n^{\circ} 5 \text { (Julio 1935) - n³7 (Julio, 1943) }\end{array}$ \\
\hline & & $\begin{array}{c}\text { Humberto Díaz Vera } \\
n^{\circ} 38-39 \text { (Octubre 1943-Enero 1944) - n } 57 \text { (Julio 1948) }\end{array}$ \\
\hline & & $\begin{array}{c}\text { Luis Bisquertt Susarte } \\
n^{\circ} 58 \text { (Diciembre 1948) - n } 66 \text { (Octubre 1950) }\end{array}$ \\
\hline \multirow{2}{*}{$\begin{array}{l}\text { Revista Chilena de } \\
\text { Educación Física } \\
\text { 1951-1969 }\end{array}$} & \multirow{2}{*}{$\begin{array}{l}\text { Instituto de Educación Física y } \\
\text { Técnica de la Universidad de } \\
\text { Chile }\end{array}$} & $\begin{array}{c}\text { Luis Bisquertt Susarte } \\
n^{\circ} 67 \text { (Enero 1951) - n } 129 \text { (Julio 1966) }\end{array}$ \\
\hline & & $\begin{array}{c}\text { Rosauro Salas Arellano } \\
n^{\circ} 130 \text { (Octubre 1966) - } n^{\circ} 139-140-141-142 \text { (1969) }\end{array}$ \\
\hline \multirow{9}{*}{$\begin{array}{c}\text { Educación Física- } \\
\text { Chile } \\
1974-2013\end{array}$} & \multirow{9}{*}{$\begin{array}{l}\text { Departamento de Educación } \\
\text { Física de la Universidad de } \\
\text { Chile / DEFDER, Universidad de } \\
\text { Chile / DEFDER, UMCE }\end{array}$} & $\begin{array}{c}\text { Rosauro Salas Arellano } \\
n^{\circ} 143 \text { (Noviembre 1974) - } n^{\circ} 222 \text { (Septiembre 1990) }\end{array}$ \\
\hline & & $\begin{array}{c}\text { Sergio Guarda Etcheverry } \\
n^{\circ} 223 \text { (Diciembre 1990) - n² } 229 \text { (Diciembre 1992) }\end{array}$ \\
\hline & & $\begin{array}{c}\text { Gabriel Figueroa Saavedra } \\
n^{\circ} 230 \text { (Mayo 1993) - n² } 247 \text { (Diciembre 1998) }\end{array}$ \\
\hline & & $\begin{array}{c}\text { Raúl Santana Núñez } \\
\text { n²48 (Mayo 1999) - n² } 256 \text { (Noviembre 2001) }\end{array}$ \\
\hline & & $\begin{array}{c}\text { Gabriel Figueroa Saavedra } \\
n^{\circ} 257 \text { (Mayo 2002) - n²66 (Diciembre 2007) }\end{array}$ \\
\hline & & $\begin{array}{c}\text { Raúl Santana Núñez } \\
\text { n²67 (Diciembre 2008) - n² } 268 \text { (Diciembre 2009) }\end{array}$ \\
\hline & & María Angélica Vergara (2011) \\
\hline & & Gloria Astudillo Rubio (2012) \\
\hline & & Marcelo González Orb (2013) \\
\hline
\end{tabular}


Respecto al número de artículos publicados, estos ascendieron a 1902. El Gráfico 1 muestra su evolución temporal, en la que se observa un comportamiento irregular en todo el periodo estudiado así como en cada etapa de la revista. La productividad anual de la revista alcanzó su máximo en 1930, con 145 artículos, y su mínimo en el 2006 con tan solo dos.

Gráfico 1 - Distribución anual de artículos publicados en la revista Educación Física-Chile (1929-2013)

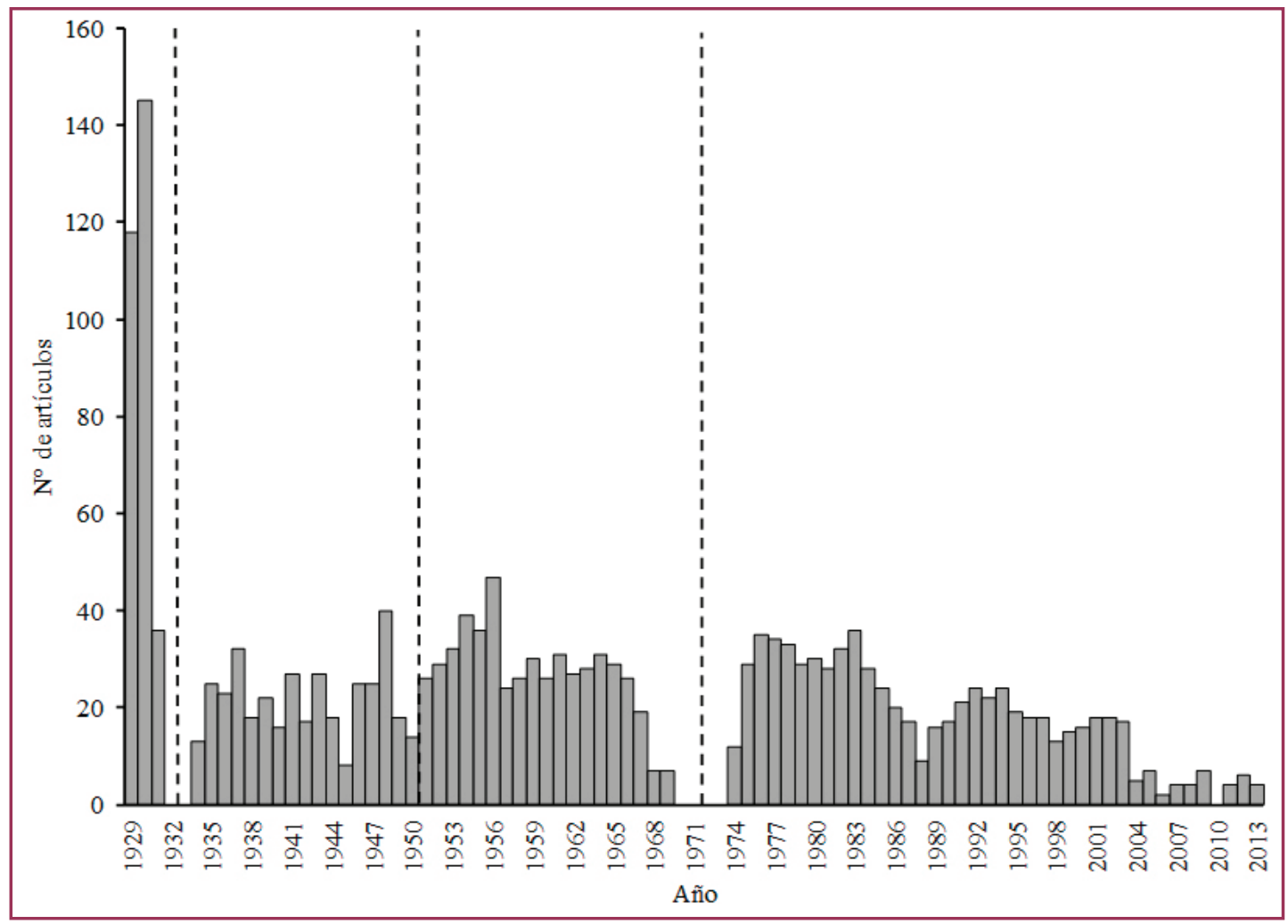

Fuente: Elaboración propia.

Los artículos publicados correspondieron a 21 disciplinas diferentes, que se agruparon en cuatro grandes ramas de conocimiento (Tabla 1). Dentro de las disciplinas, destaca la Teoría de la Educación Física y el Deporte, concentrando el 31.6\% del total de artículos y en segundo término siete disciplinas más que superan los 100 artículos $(80.5 \%$ de los artículos totales). Respecto a las ramas de conocimiento, destaca la de Ciencias Sociales (48,37\% del total de artículo). Al analizar cada fase de la revista, se observa un predominio de las Ciencias Sociales y las Ciencias de la Vida sobre el resto, mostrando ambas un crecimiento progresivo en las dos últimas etapas de la revista. Por el contrario, Artes y Humanidades y Tecnología presentaron un ligero crecimiento en el número de artículos hasta 1969, para luego disminuir.

En Educación Física-Chile participaron un total de 992 autores, pertenecientes a 255 instituciones de 38 países diferentes (Tabla 2). En términos absolutos el número de autores e instituciones creció a lo largo de las etapas de la revista, no así el número de países representados, que disminuyó en la última etapa. No obstante, en términos relativos (autores/ año) los resultados muestran un panorama diferente, con 44,3 autores/año en el primer periodo, 11,6 en el segundo, 17,6 en el tercero y 10,1 en el último periodo. 
Tabla 1 - Distribución de artículos en la revista Educación Física-Chile según su rama, disciplina y periodo de publicación

\begin{tabular}{|c|c|c|c|c|c|c|c|c|c|c|}
\hline \multirow{2}{*}{ Ramas y disciplina } & \multicolumn{2}{|c|}{ Total } & \multicolumn{2}{|c|}{$1929-1931$} & \multicolumn{2}{|c|}{ 1934-1950 } & \multicolumn{2}{|c|}{ 1951-1969 } & \multicolumn{2}{|c|}{$1974-2013$} \\
\hline & $n$ & $\%$ & $n$ & $\%$ & $N$ & $\%$ & $n$ & $\%$ & $n$ & $\%$ \\
\hline Artes y Humanidades & 215 & 11,3 & 20 & 1,05 & 51 & 2,68 & 81 & 4,26 & 63 & 3,31 \\
\hline Arquitectura & 2 & 0,11 & 1 & 0,05 & & & & & 1 & 0,05 \\
\hline Filosofía & 1 & 0,05 & 1 & 0,05 & & & & & & \\
\hline Historia & 212 & 11,15 & 18 & 0,95 & 51 & 2,68 & 81 & 4,26 & 62 & 3,26 \\
\hline Ciencias de la Vida & 635 & 33,39 & 99 & 5,21 & 113 & 5,94 & 180 & 9,46 & 243 & 12,78 \\
\hline Actividad física y salud & 24 & 1,26 & 4 & 0,21 & 1 & 0,05 & 6 & 0,32 & 13 & 0,68 \\
\hline Antropología & 1 & 0,05 & & & & & 1 & 0,05 & & \\
\hline Antropometría & 26 & 1,37 & 1 & 0,05 & 7 & 0,37 & 3 & 0,16 & 15 & 0,79 \\
\hline Biomecánica & 56 & 2,94 & 8 & 0,42 & 13 & 0,68 & 13 & 0,68 & 22 & 1,16 \\
\hline Cinesiología & 22 & 1,16 & 1 & 0,05 & 4 & 0,21 & 16 & 0,84 & 1 & 0,05 \\
\hline Fisiología & 202 & 10,62 & 19 & 1 & 53 & 2,79 & 59 & 3,1 & 71 & 3,73 \\
\hline Medicina del Deporte & 109 & 5,73 & 33 & 1,74 & 17 & 0,89 & 29 & 1,52 & 30 & 1,58 \\
\hline Nutrición & 39 & 2,05 & 5 & 0,26 & 11 & 0,58 & 14 & 0,74 & 9 & 0,47 \\
\hline $\begin{array}{l}\text { Teoría del Entrenamiento } \\
\text { Deportivo }\end{array}$ & 156 & 8,2 & 28 & 1,47 & 7 & 0,37 & 39 & 2,05 & 82 & 4,31 \\
\hline Ciencias Sociales & 920 & 48,37 & 164 & 8,62 & 166 & 8,73 & 209 & 10,99 & 381 & 20,03 \\
\hline Derecho & 1 & 0,05 & & & & & & & 1 & 0,05 \\
\hline Didáctica & 135 & 7,1 & 22 & 1,16 & 31 & 1,63 & 27 & 1,42 & 55 & 2,89 \\
\hline Psicología & 82 & 4,31 & 5 & 0,26 & 14 & 0,74 & 12 & 0,63 & 51 & 2,68 \\
\hline Psicomotricidad & 38 & 2 & & & & & 5 & 0,26 & 33 & 1,74 \\
\hline Sociología & 63 & 3,31 & 4 & 0,21 & 7 & 0,37 & 27 & 1,42 & 25 & 1,31 \\
\hline $\begin{array}{l}\text { Teoría de la Educación Física } \\
\text { y el Deporte }\end{array}$ & 601 & 31,6 & 133 & 6,99 & 114 & 5,99 & 138 & 7,26 & 216 & 11,36 \\
\hline Tecnología & 132 & 6,94 & 16 & 0,84 & 38 & 2,00 & 50 & 2,63 & 28 & 1,47 \\
\hline Documentación & 15 & 0,79 & & & 5 & 0,26 & 3 & 0,16 & 7 & 0,37 \\
\hline Gestión & 116 & 6,1 & 16 & 0,84 & 33 & 1,74 & 47 & 2,47 & 20 & 1,05 \\
\hline Informática & 1 & 0,05 & & & & & & & 1 & 0,05 \\
\hline Total & 1902 & 100 & 299 & 15,72 & 368 & 19,35 & 520 & 27,34 & 715 & 37,59 \\
\hline
\end{tabular}

Tabla 2 - Distribución de autores, instituciones y países representados en la revista Educación Física-Chile según sus etapas

\begin{tabular}{lcccccccccc}
\hline \multirow{2}{*}{ Variable } & \multicolumn{2}{c}{ Total } & \multicolumn{2}{c}{$1929-1931$} & \multicolumn{2}{c}{$1934-1950$} & \multicolumn{1}{c}{$1951-1969$} & \multicolumn{2}{c}{$1974-2013$} \\
\cline { 2 - 10 } & $n$ & $\%$ & $n$ & $\%$ & $n$ & $\%$ & $n$ & $\%$ & $n$ & $\%$ \\
\hline Autores & 992 & 100 & 133 & 13,4 & 197 & 19,9 & 335 & 33,8 & 403 & 40,6 \\
Instituciones & 255 & 100 & 18 & 7,1 & 75 & 29,4 & 95 & 37,3 & 105 & 41,2 \\
Países & 38 & 100 & 8 & 21,1 & 18 & 47,4 & 32 & 84,2 & 17 & 44,7 \\
\hline
\end{tabular}

n: frecuencia; \%: respecto al total de autores (992), instituciones (255) y países (38) respectivamente.

Fuente: Elaboración propia.

La Tabla 3 muestra las instituciones que aparecen representadas con más de 10 firmas en Educación Física-Chile. La revista fue principalmente el órgano de difusión de trabajos 
desarrollados por los profesores del DEFDER $(40,06 \%$ del total), con una representación especialmente relevante en el periodo 1974-2013. Un 32,64\% de las firmas omitían la adscripción institucional de su autor, agrupando entre ambas el $72,7 \%$ del total. El resto de instituciones no superaron las 30 firmas.

Tabla 3 - Distribución de firmas por institución en la revista Educación Física-Chile según sus etapas

\begin{tabular}{|c|c|c|c|c|c|c|c|c|c|c|}
\hline \multirow{2}{*}{ Institución } & \multicolumn{2}{|c|}{ Total } & \multicolumn{2}{|c|}{$1929-1931$} & \multicolumn{2}{|c|}{$1934-1950$} & \multicolumn{2}{|c|}{ 1951-1969 } & \multicolumn{2}{|c|}{$1974-2013$} \\
\hline & $n$ & $\%$ & $n$ & $\%$ & $n$ & $\%$ & $n$ & $\%$ & $n$ & $\%$ \\
\hline DEFDER & 945 & 40,06 & 8 & 0,34 & 251 & 10,64 & 120 & 5,09 & 566 & 23,99 \\
\hline Desconocida & 770 & 32,64 & 265 & 11,23 & 54 & 2,29 & 252 & 10,68 & 199 & 8,44 \\
\hline U. de Tarapacá & 28 & 1,19 & & & & & & & 28 & 1,19 \\
\hline $\begin{array}{l}\text { U. de } \\
\text { Concepción }\end{array}$ & 26 & 1,10 & & & 1 & 0,04 & 14 & 0,59 & 11 & 0,47 \\
\hline $\begin{array}{l}\text { Instituto Central } \\
\text { de Gimnasia } \\
\text { de Estocolmo } \\
\text { (Suecia) }\end{array}$ & 19 & 0,81 & & & 18 & 0,76 & 1 & 0,04 & & \\
\hline U. de Chile & 19 & 0,81 & & & 1 & 0,04 & 9 & 0,38 & 9 & 0,38 \\
\hline $\begin{array}{l}\text { Desconocida } \\
\text { (Argentina) }\end{array}$ & 18 & 0,76 & 1 & 0,04 & 5 & 0,21 & 8 & 0,34 & 4 & 0,17 \\
\hline $\begin{array}{l}\text { Comisión } \\
\text { Nacional de } \\
\text { Educación } \\
\text { Física (Uruguay) }\end{array}$ & 15 & 0,64 & 2 & 0,08 & 4 & 0,17 & 9 & 0,38 & & \\
\hline $\begin{array}{l}\text { Instituto de } \\
\text { Educación } \\
\text { Física (Uruguay) }\end{array}$ & 15 & 0,64 & & & 3 & 0,13 & 12 & 0,51 & & \\
\hline $\begin{array}{l}\text { U. de Granada } \\
\text { (España) }\end{array}$ & 14 & 0,59 & & & & & & & 14 & 0,59 \\
\hline $\begin{array}{l}\text { U. de Lieja } \\
\text { (Bélgica) }\end{array}$ & 13 & 0,55 & & & 4 & 0,17 & 9 & 0,38 & & \\
\hline $\begin{array}{l}\text { U. de lowa (EE. } \\
\text { UU.) }\end{array}$ & 13 & 0,55 & & & 3 & 0,13 & 10 & 0,42 & & \\
\hline
\end{tabular}

Academia

Superior de

$\begin{array}{lllll}\text { Ciencias } & 12 & 0,51 & 12 & 0,51\end{array}$

Pedagógicas de

Santiago

Pontificia U.

$\begin{array}{lllll}\text { Católica de } & 11 & 0,47 & 11 & 0,47\end{array}$

Valparaíso

\begin{tabular}{lcccccccccc}
\hline UMCE & 11 & 0,47 & & & & & & & 11 & 0,47 \\
\hline Otras & 430 & 18,23 & 25 & 1,06 & 84 & 3,56 & 120 & 5,09 & 201 & 8,52 \\
\hline Total & $\mathbf{2 3 5 9}$ & $\mathbf{1 0 0}$ & $\mathbf{3 0 1}$ & $\mathbf{1 2 , 7 6}$ & $\mathbf{4 2 8}$ & $\mathbf{1 8 , 1 4}$ & $\mathbf{5 6 4}$ & $\mathbf{2 3 , 9 1}$ & $\mathbf{1 0 6 6}$ & $\mathbf{4 5 , 1 9}$ \\
\hline
\end{tabular}

n: frecuencia; \%: respecto al total de firmas (2359).

Fuente: Elaboración propia.

Aunque la distribución de firmas según país muestra una amplia participación de autores chilenos (50,61\% del total) y desafortunadamente el 33,66\% de las firmas no incluyeron su país de afiliación, se registraron un total de 38 países distintos, destacando España y Argentina 
(Tabla 4). A pesar de las diferencias idiomáticas, también colaboraron autores suecos, belgas, estadounidenses, brasileños, alemanes o franceses, entre otros.

Tabla 4 - Distribución de firmas por país en la revista Educación Física-Chile según sus etapas

\begin{tabular}{|c|c|c|c|c|c|c|c|c|c|c|}
\hline \multirow{2}{*}{ País } & \multicolumn{2}{|c|}{ Total } & \multicolumn{2}{|c|}{$1929-1931$} & \multicolumn{2}{|c|}{ 1934-1950 } & \multicolumn{2}{|c|}{$1951-1969$} & \multicolumn{2}{|c|}{$1974-2013$} \\
\hline & $n$ & $\%$ & $n$ & $\%$ & $n$ & $\%$ & $n$ & $\%$ & $n$ & $\%$ \\
\hline Chile & 1194 & 50,61 & 22 & 0,93 & 281 & 11,91 & 159 & 6,74 & 732 & 31,03 \\
\hline Desconocido & 794 & 33,66 & 268 & 11,36 & 57 & 2,42 & 260 & 11,02 & 209 & 8,86 \\
\hline España & 51 & 2,16 & 1 & 0,04 & 1 & 0,04 & 1 & 0,04 & 48 & 2,03 \\
\hline Argentina & 50 & 2,12 & 1 & 0,04 & 14 & 0,59 & 22 & 0,93 & 13 & 0,55 \\
\hline Uruguay & 34 & 1,44 & 3 & 0,13 & 9 & 0,38 & 22 & 0,93 & & \\
\hline EE.UU. & 32 & 1,36 & & & 10 & 0,42 & 22 & 0,93 & & \\
\hline Suecia & 32 & 1,36 & & & 27 & 1,14 & 5 & 0,21 & & \\
\hline Bélgica & 29 & 1,23 & & & 13 & 0,55 & 15 & 0,64 & 1 & 0,04 \\
\hline Brasil & 22 & 0,93 & & & 1 & 0,04 & 4 & 0,17 & 17 & 0,72 \\
\hline Alemania & 21 & 0,89 & 4 & 0,17 & & 0,00 & 6 & 0,25 & 11 & 0,47 \\
\hline Francia & 17 & 0,72 & 1 & 0,04 & 2 & 0,08 & 9 & 0,38 & 5 & 0,21 \\
\hline Cuba & 14 & 0,59 & & & 1 & 0,04 & 1 & 0,04 & 12 & 0,51 \\
\hline Otros & 69 & 2,92 & 1 & 0,04 & 12 & 0,51 & 38 & 1,61 & 18 & 0,76 \\
\hline Total & 2359 & 100 & 301 & 12,8 & 428 & 18,1 & 564 & 23,9 & 1066 & 45,2 \\
\hline
\end{tabular}

\section{DISCUSIÓN}

Educación Física-Chile comenzó como el órgano oficial de la Dirección General de Educación Física, estando al cargo el Teniente Osvaldo Kolbach, y como responsable de la revista Rosauro Salas Arellano, aunque no hay duda que su afiliación era el Instituto de Educación Física, que al depender de dicha Dirección tomó el nombre de Escuela de Profesores de Educación Física (SALAS, 1989). La primera editorial no solo mostraba la variedad documental que incluiría sino también la responsabilidad encomendada por la Dirección General de Educación Física:

Los profesores del ramo encontrarán una escogida selección de artículos científicos, crónicas que detallen los más importantes acontecimientos deportivos, nacionales o extranjeros y bibliografías de las obras más interesantes de los médicos y maestros que consagraron sus desvelos al progreso de la Educación Física.

La voz oficial, manifestada por medio de circulares o notas, tendrá una sección exclusiva, la que unida a las encuestas que haremos, determinarán los rumbos científicos que han de guiar a los que hemos recibido la notable misión de fomentar la Educación Física, de tal suerte que, formando hábitos en todas las clases sociales, llegue a ser en cada ciudadano una cuotidiana e imprescindible necesidad. (NUESTRO..., 1929, p. 1)

Con una duración de tres años, la fuerza de la revista en esta primera etapa fue innegable: se publicaron 299 artículos (99,7 artículos/año) por 133 autores de 18 instituciones y ocho países, teniendo una periodicidad mensual (desafortunadamente la mayoría de artículos 
no indicaban la afiliación de sus autores por lo que resulta imposible conocer las instituciones o países con mayor aportación a la revista). Desde estos inicios, ya se puede apreciar el carácter multidisciplinar de Educación Física-Chile, que le acompañaría a lo largo de su historia, así como su mayor tendencia hacia la rama de conocimiento de las Ciencias Sociales y la disciplina de Teoría de la Educación Física y el Deporte. El descenso de publicaciones del año 1931 se debió a que el último número de esta etapa se publicó en abril de 1931. Aunque Guarda Etcheverry (2006a) menciona que esta primera etapa finalizó en 1933 sin ofrecer más datos sobre la misma, el trabajo de Salas (1989) confirma la fecha de finalización en 1931, añadiendo que fueron razones económicas las que hicieron desaparecer a la revista. Efectivamente, las consecuencias de la Depresión norteamericana provocaron una grave crisis económica en Chile (COLLIER; SATER, 1999) que afectó a todos los ámbitos. A esta situación se añadió la inestabilidad política existente entre 1931 y 1932 (COLLIER; SATER, 1999, SILVA GALDAMES, 1995), siendo un ejemplo de este desorden los trece ministros que tuvo el Ministerio de Educación en dicho periodo (COX et al., 1997).

En su segunda etapa (1934-1950), iniciada tras dos años de suspensión, la revista reapareció con el título Boletín de Educación Física. En su primer editorial se dibujaba el panorama de cambios que estaba viviendo la educación física, y que darían sentido a la publicación:

El gran progreso experimentado por las Ciencias Biológicas y los avances que ha hecho la Pedagogía Científica en los últimos años, han repercutido lógicamente en las orientaciones de la Educación Física y han planteado nuevos e interesantes problemas a quienes con ella se ocupan. (NOTAS..., 1934, p. 1)

Junto a las razones económicas y políticas que habían motivado su suspensión en 1931, debe mencionarse el cambio de adscripción y la reestructuración del Instituto que pasó a depender del Ministerio de Guerra en 1931 y después de la Universidad de Chile tras su reestructuración (COFRÉ ILUFFI, 1989, CORNEJO AMÉSTICA; MATUS CASTILLO; VARGAS CONTRERAS, 2011, RUIZ URBINA, 1956). Una vez restaurada, en sus 17 años de existencia publicó 368 artículos, firmados por 197 autores de 75 instituciones y 18 países. En relación a la variedad disciplinar de la revista, en esta etapa se siguió, en términos generales, la tónica de la anterior. La importancia de estos datos en términos absolutos, sin embargo, no esconde el hecho de los altibajos de la revista en esta etapa, que se refleja en la breve permanencia de dos de sus cuatro directores en su puesto, o en la irregularidad del número de artículos publicados por año, que osciló entre ocho en 1945, y 40 en 1948, solo tres años después.

Para su tercera etapa, tal como indicaba el director de la revista Luis Bisquertt en las notas editoriales, era necesario cambiar la denominación de la revista debido a su prestigio y para mostrar mejor la trascendencia, finalidad y tradición de la misma (NOTAS..., 1951). Ahora, con el nombre de Revista Chilena de Educación Física se inició un periodo en el que se publicaron 520 artículos por 335 autores de 95 instituciones y 32 países, lo cual muestra una mayor apertura de la revista que en la etapa precedente. Asimismo, sigue su línea multidisciplinar, y aparece la psicomotricidad como una nueva disciplina de la educación física. Esta buena situación de la revista se mantendría hasta la segunda mitad de la década de 1960, en la que se produce un descenso en picado hasta 1970 que desaparece nuevamente. En este sentido, desde 1960 y hasta 1973 las universidades se vieron afectadas por la conocida "reforma universitaria" que conllevó no solo transformaciones en sus contenidos, orientaciones 
y estructuras sino también importantes conflictos y tensiones con el movimiento estudiantil (AGÜERO, 1985, GARRETÓN; MARTÍNEZ, 1985, HUNEEUS, 1988). Tras una primera fase de gestación, es a partir de 1965 cuando comenzó a producirse la reforma del sistema universitario; empezando con el endurecimiento de las relaciones entre los estudiantes y las autoridades universitarias, continuando con la aplicación de las medidas de transformación a partir de 1967 y finalizando con la consolidación parcial de un nuevo modelo de universidad a partir de 1970 (CASALI FUENTES, 2011, GARRETÓN; MARTÍNEZ, 1985). Sin duda esta reforma universitaria afectó al funcionamiento del sistema universitario en general y al de la Universidad de Chile en particular, explicando la desaparición de la revista desde 1970 hasta 1973. Además, tras el golpe militar del 11 de septiembre de 1973 las universidades fueron intervenidas militarmente y se procedió a la destitución de autoridades y la eliminación de sectores docentes, estudiantiles, administrativos, así como centros universitarios especialmente en el área de Ciencias Sociales (GARRETÓN; MARTÍNEZ, 1985), lo que explica que tampoco se editara con normalidad en 1974. Todo ello tuvo repercusiones directas en el Instituto de Educación Física y Técnica, con las limitaciones presupuestarias que le afectaron y que provocaron, en primer lugar, que solo apareciera un número de la revista en 1968 y 1969 y, en segundo lugar, que desapareciera desde 1970 hasta 1974 (GUARDA ETCHEVERRY, 2006a). Además, el Instituto cambió su sede a la comuna de Ñuñoa en 1969, y pasó a depender de la Facultad de Filosofía y Educación en 1969, del Departamento de la Sede Oriente en 1972 y de la Facultad de Educación en 1974 (GUARDA ETCHEVERRY, 2006b).

Tras esta segunda crisis, el aporte financiero de la Dirección General de Deportes y Recreación (GUARDA ETCHEVERRY, 2006a) permitió que la revista, ahora Educación FísicaChile reiniciase su andadura en 1974. Durante esta etapa, solo interrumpida en el año 2010, la revista ha dependido del DEFDER adscrito hasta el año 1985 a la Universidad de Chile y posteriormente a la UMCE. Hasta el año 2013 se publicaron, en un mismo ámbito multidisciplinar que en etapas anteriores, 715 artículos por 403 autores de 105 instituciones de 17 países, número que en términos relativos muestran un empeoramiento de la situación de la revista. Después de una primera fase de crecimiento y estabilización hasta 1983, con una producción media de 30 artículos/año, la revista experimentó un descenso progresivo y una fase de altibajos hasta el año 2003. A partir de entonces la periodicidad de la revista fue anual, lo que redujo considerablemente el número de artículos publicados. Esta tendencia decreciente es totalmente inversa respecto al desarrollo de las CCAFD en el ámbito internacional (BIRRELL, 2006) y en Chile durante las últimas décadas. Particularmente en Chile, los resultados de Andrade et al. (2013) presentaron una evolución creciente de la producción científica de Ciencias del Deporte indexada en ISI Web of Knowledge en Sudamérica y en Chile desde 1970 hasta 2013. Igualmente, el aumento del número de instituciones impartiendo estudios relacionados con las CCAFD, de 12 en 1989 (COFRÉ ILUFFI, 1989) a más de 30 en la actualidad (CONSEJO ACADÉMICO NACIONAL DE EDUCACIÓN FÍSICA, 2015); y la progresiva aparición de otras revistas especializadas en 1993 (REVISTA CIENCIAS DE LA ACTIVIDAD FÍSICA, 2015), 1998 (MONTECINOS ESPINOZA, 1998), 2001 (MOTRICIDAD HUMANA, 2015), 2004 (VELASCO RODRÍGUEZ, 2004), 2006 (MOTRICIDAD Y PERSONA, 2015) y 2010 (HORIZONTE, 2015), representan un crecimiento de las CCAFD en Chile. Esta situación debería interpretarse como un entorno favorable aunque más competitivo al que Educación Física-Chile no está sabiendo dar respuesta. Su periodicidad anual desde 2004, la desaparición de 2010 y los sucesivos cambios en su dirección durante los últimos tres años parecen indicar, si no la posible desaparición de 
esta longeva publicación, sí su situación marginal en el panorama actual de publicaciones de CCAFD, ya que no está indexada en bases de datos como Scielo, Scopus o Web of Science y no cuenta con una plataforma propia de acceso abierto a sus artículos en formato digital.

\section{CONCLUSIÓN}

Durante sus cuatro etapas, Educación Física-Chile ha sido un importante medio para la difusión de las investigaciones relacionadas con el conjunto de las CCAFD en Chile. Aunque este trabajo ha centrado su análisis sobre artículos y revisiones, existen otro tipo de documentos (notas, crónicas, editoriales,...) y secciones (actualidad, bibliográfica,...) que refuerzan el carácter multidisciplinar y la importancia de esta publicación para el estudio de la educación física chilena.

Educación Física-Chile sufrió especialmente las consecuencias en Chile de la Depresión norteamericana y la inestabilidad política entre 1931-1932, así como la reforma universitaria y el golpe militar entre 1965-1974. A pesar de estos problemas, el esfuerzo de numerosas personas e instituciones, particularmente el DEFDER, ha posibilitado que esta revista siga editándose. No obstante, desde la década de 1990 Educación Física-Chile está experimentando una marcada involución que contrasta con el crecimiento de las CCAFD a nivel global y en Chile, y que la sitúan actualmente en una situación de marginalidad y potencial riesgo de desaparición.

\section{REFERENCIAS}

AENOR, Agencia Española de Normalización. UNE-ISO 690-2013. Información y documentación. Directrices para la redacción de referencias bibliográficas y de citas de recursos de información. Madrid: Agencia Española de Normalización, 2013.

AGÜERO, Felipe. La reforma en la Universidad de Chile. Santiago: Ediciones Sur, 1985.

ANDRADE, David Cristóbal et al. Bibliometric analysis of South American research in sports science from 1970 to 2012. Motriz, Rio Claro, v. 19, n. 4, p. 783-791, 2013.

ARBINAGA IBARZÁBAL, Félix; ARAGÓN DOMÍNGUEZ, Juan D.; TEJEDOR BENITTEZ, Rocío. Análisis bibliométrico de la Revista de Psicología del Deporte (1992-2009). Revista de Psicología del Deporte, Palma, v. 19, n. 2, p. 231-245, 2010.

BIRRELL, Susan. Sport and Sport Studies. In: ROJEK, Chris; SHAW, Susan; VEAL, Tony (Ed.). The Handbook of leisure studies. London: Palgrave Macmillan, 2006. p. 335-353.

CASALI FUENTES, Aldo. Reforma universitaria en Chile, 1967-1973: pre-balance histórico de una experiencia frustrada. Intus-Legere Historia, Santiago, v. 5, n. 1, p. 81-101, 2011.

CHILE. Ministerio de Educación Buscador de carreras. 2015. Disponível em: <http://mifuturo.cl/ index.php/donde-y-que-estudiar/buscador-de-carreras>. Acesso em: 6 abr. 2015.

COFRÉ ILUFFI, Milton. El DEFDER y sus 80 años: el Instituto Superior de Educación Física y Técnica, hoy Departamento de Educación Física, Deportes y Recreación. Educación FísicaChile, Santiago, v. 57, n. 208, p. 12-15, 1986. 
COFRÉ ILUFFI, Milton. El Instituto de Educación Física y Manual: ocho décadas. Educación Física-Chile, Santiago, v. 61, n. 219, p. 9-19, 1989.

COLLIER, Simon; SATER, William F. Historia de Chile: 1808-1994. Madrid: Cambridge University, 1999.

CONSEJO ACADÉMICO NACIONAL DE EDUCACIÓN FÍSICA. Universidades del Consejo. Disponível em: <http://www.canef.cl/\#!consejo/c3qn>. Acesso em: 6 abr. 2015.

CORNEJO AMÉSTICA, Miguel; MATUS CASTILLO, Carlos; VARGAS CONTRERAS, Camilo. La Educación Física en Chile: una aproximación histórica. Lecturas: Educación fisica y deportes, Buenos Aires, v. 16, n. 161, 2011.

COX, Cristián et al. 160 años de educación pública: historia del Ministerio de Educación. Santiago: Ministerio de Educación, 1997.

CROXATTO, Héctor. La revista "Educación Física, Chile". Educación Física-Chile, Santiago, v. 57, n. 208, p. 11, 1986.

DEVÍS DEVÍS, José et al. Disciplinas y temas de estudio en las ciencias de la actividad física y el deporte. Revista Internacional de Medicina y Ciencias de la Actividad Física y del Deporte, Madrid, v.10, n. 37, p. 150-166, 2010.

GARRETÓN, Manuel Antonio; MARTÍNEZ, Javier. Universidades chilenas: historia, reforma e intervención. Santiago: SUR, 1985.

GUARDA ETCHEVERRY, Sergio. Historia de la Revista de Educación Física. Educación Física Chile, Santiago, n. 265, p. 41-45, 2006a.

GUARDA ETCHEVERRY, Sergio. Historia del Instituto de Educación Física. Educación física Chile, Santiago, n. 265, p. 13-24, 2006b.

HENNESSEY, Christina. A Bibliometric Analysis of the Journal of Sport History. In: LOMAX, M. E. (Ed). ANNUAL NASSH CONFERENCE, 32nd., 2004. Proceedings... Athens: Sadiki Publishing, 2004. p. 55-56

HORIZONTE. Revista Horizonte: Ciencias de la actividad física. 2015. Disponível em: <http:// revistahorizonte.ulagos.cl/>. Acesso em: 6 abr. 2015.

HUNEEUS, Carlos. La reforma universitaria: veinte años después. Santiago: Corporación de Promoción Universitaria, 1988.

LÓPEZ MORENO, Alberto; VELASCO MARTÍN, Alfonso. Breve análisis de la producción científica de la revista Archivos de medicina del deporte: periodo 1984-2002. Archivos de Medicina del Deporte, Pamplona, v. 21, n.100, p. 93-98, 2004.

MONTECINOS ESPINOZA, Roberto. Presentación. Ciencias de la Actividad Física U.C.M., Talca, v. 1, n. 1, p. 5, 1998.

MOTRICIDAD HUMANA. Sobre la revista. 2015. Disponível em: <http://www.revistamotricidad. com/?page_id=254>. Acesso em: 6 abr. 2015.

MOTRICIDAD Y PERSONA. Presentación revista Motricidad y Persona. Disponível em: $<$ http://www.ucentral.cl/revista-motricidad-y-persona/prontus_ucentral2012/2012-01-03/164025. html>. Acesso em: 6 abr. 2015. 
NEVILL, Alan; ATKINSON, Greg; HUGHES, Mike. Twenty-five years of sport performance research in the Journal of Sports Sciences. Journal of Sports Sciences, v. 26, n. 4, p. 413-426, 2008.

NOTAS editoriales: nuestro boletín. Boletín de Educación Física, Santiago, v. 1, n. 1, 1934.

NOTAS editoriales: nuevo nombre. Revista Chilena de Educación Física, Santiago, v. 67, n. 2 , 1951.

NUESTRO programa. Educación Física, Santiago, v. 1, n. 1, 1929.

PERRINO PEÑA, María. Análisis bibliométrico, temático e ideológico de la revista de estudios deportivos Citius, Altius, Fortius (1959-1976). León: Universidad de León, 2014.

PITTS, Brenda; DANYLCHUK, Karen; QUARTERMAN, Jerome. Analysis of Sport Management Literature: European Sport Management Quarterly. Sport Management International Journal, Sparta, v. 20, n. 2, p. 45-72, 2014.

POBLETE GÁLVEZ, Carolina Alejandra. La construcción del conocimiento histórico de la Educación Física en Chile. Una mirada a la primera revista latinoamericana en la especialidad durante los años comprendidos entre 1934 y 1962. 413 f. Tesis (Doctoral) - Universidad de Granada, Granada, 2014.

REVISTA CIENCIAS DE LA ACTIVIDAD FÍSICA. 2015. Disponível em: <http://www.upla.cl/ actividadfisica/publicaciones/>. Acesso em: 6 abr. 2015.

RUBILAR SOLIS, Luis. Universidad Metropolitana de Ciencias de la Educación (ExPedagógico), 1989-2010. [s.I.]: Publicaciones UMCE, 2012.

RUIZ URBINA, Antonio. Bosquejo histórico del Instituto de Educación Física y Técnica de la Universidad de Chile. Revista Chilena de Educación Física, v. 22, n.90, p.1523-1599, 1956.

SALAS, Rosauro. 60 años de una revista: Educación Física, Chile. Educación Física-Chile, Santiago, v. 60, n. 218, p. 4-8, 1989.

SILVA GALDAMES, Osvaldo. Breve historia contemporánea de Chile. Santiago: Fondo de Cultura Económica, 1995.

THOMSON REUTERS. Research areas. 2012. Disponível em: <http://images.webofknowledge. com/WOKRS57B4/help/WOS/hp research areas easca.html>. Acesso em: 12 abr. 2015.

VELASCO RODRÍGUEZ, Ernesto. Editorial. Revista de Ciencias de la Actividad Física y Deportes, v. 1, n. 1, p. 5, 2004.

WILLIAMS, A. Mark; HARDY, Lew; MUTRIE, Nanette. Twenty-five years of psychology in the Journal of Sports Sciences: A historical overview. Journal of Sports Sciences, London, v.26, n.4, p.401-412, 2008. 